\title{
Beta condicional para liquidez no mercado acionário brasileiro e americano
}

\author{
Conditional beta for liquidity in brazilian and us stock market
}

\author{
Vinicius Girardi da Silveira ${ }^{1}$ \\ Rodrigo Abbade da Silva ${ }^{2}$ \\ Kelmara Mendes Vieira ${ }^{3}$ \\ Raphael Silveira Amaro ${ }^{4}$ \\ Diego Antonio Bittencourt Marconatto ${ }^{5}$
}

\begin{abstract}
RESUMO
A proposta deste estudo é examinar a existência de um prêmio pelo risco ligado à falta de liquidez nos mercados de ações brasileiro e norte-americano. Inicialmente, foi estimada a matriz de variância-covariância para os retornos e volumes de ambos os mercados utilizando o modelo Copula-DCC-GARCH. Depois disso, foram utilizadas estimações para criar um índice beta condicional para liquidez. Este estudo confirma a hipótese de um prêmio pelo risco devido à falta de liquidez apenas para o mercado norte-americano. Por outro lado, os resultados para o mercado brasileiro mostram um prêmio durante o aumento da liquidez. Esses achados sugerem que os investidores brasileiros não recebem uma compensação para negociar ações com baixa liquidez. Este artigo é importante por que mostra uma relação financeira inesperada ao longo do tempo para um mercado de ações latino. Posteriormente, pode ser feito um elo entre os custos de transação e a teoria financeira para explicar esse fenômeno.
\end{abstract}

Palavras-chave: Mercado de ações. Liquidez. Índice Beta Condicional. Teoria dos custos de transação.

\begin{abstract}
The purpose of this paper is to examine the existence of a risk premium linked to the lack of liquidity in the US and Brazilian stock markets. As the first step, were estimated the variance-covariance matrix for the returns and volumes of both markets using a Copula-DCC-GARCH model. After that, were used the estimations to create a conditional beta index for liquidity. This study confirms the hypothesis of a risk premium for the lack of liquidity just in the US market. On the other hand, the results for the Brazilian market show a premium during increases of the liquidity. These findings suggest that Brazilian investors do not receive a compensation to negotiate low liquidity stocks. This paper is important because it shows an unexpected financial relation over time for a Latin stock market. Furthermore, a link between the transaction costs and financial theory can be made to explain these phenomena.
\end{abstract}

Keywords: Stock Market. Liquidity. Conditional Beta Index. Transaction Costs Theory.

Possui Mestrado em Administração e Graduação em Ciências Econômicas pela Universidade Federal de Santa Maria. Brasil. Afiliação: Universidade Federal de Santa Maria. Lattes: http://lattes.cnpq.br/2152159888053991. Email: vinicius.girardi@hotmail.com

2 Doutorando em administração pela Universidade Federal de Santa Catarina - UFSC e Mestre em administração na Universidade Federal de Santa Maria - UFSM. Brasil. Afiliação: Universidade Federal de Santa Maria. Lattes: http://lattes.cnpq.br/5874100127589654. Email: abbaders@ gmail.com

3 Possui doutorado em Administração pela Universidade Federal do Rio Grande do Sul (2006). Professora Associada da Universidade Federal de Santa Maria. Brasil. Afiliação: Universidade Federal de Santa Maria. Lattes: http://lattes.cnpq.br/4786960732238120. Orcid: http://orcid. org/0000-0002-8847-0941. Email: kelmara@terra.com.br

4 Possui Mestrado em Administração com ênfase em Economia, Controle e Finanças pela Universidade Federal de Santa Maria (2016). Brasil. Afiliação: Universidade Federal de Santa Maria. Lattes: http://lattes.cnpq.br/8621904059935562. Email: raphael.amaro@ufsm.br

5 Pós-doutor em Administração pela HEC Montréal -- Visiting Scholar na HEC Montréal (Canadá) e na Universidad de Sevilla (Espanha). Professor Pesquisador do Programa de Pós-Graduação da Escola de Gestão e Negócios da Unisinos. Brasil. Afiliação: Universidade do Vale do Rio dos Sinos. Lattes: http://lattes.cnpq.br/9626677883636081. Email: dmarconatto@gmail.com 


\section{Introdução}

A palavra transação pode ser utilizada para representar uma interação entre diferentes pessoas que buscam negociar bens ou serviços (MILGROM; ROBERTS, 1992). Entretanto, essa negociação, por ser um fenômeno econômico, pode envolver custos, que são denominados de custos de transação. Estudos sobre esse tema tiveram origem com o surgimento da teoria econômica dos custos de transação desenvolvida por Ronald Coase no ano de 1937 (THIELMANN, 2013). O trabalho de Coase tinha como intuito compreender a dualidade existente no campo empresarial entre a escolha de produzir bens e serviços internamente ou optar pela terceirização destes. A teoria desenvolvida por Coase para examinar essa dualidade observada dentro da firma considera que a produção de um bem ou de um serviço não envolve apenas os custos de produção, mas também envolve os custos relacionados à transação.

Esses custos relacionados à transação podem ser considerados como o dispêndio de recursos econômicos para planejar e monitorar as estruturas de governança, como por exemplo, recursos gastos com a finalidade de elaborar, negociar e garantir o cumprimento de contratos (WILLIAMSON, 1985). Nesta última finalidade, percebe-se que os custos de transação seriam determinados pelas incertezas inerentes ao processo de negociação, pela frequência das operações realizadas e pelo grau de especificidade dos ativos (THIELMANN, 2013). Além desses aspectos, existem outros elementos que se relacionam de forma direta ou indireta com os custos de transação, como o oportunismo, a racionalidade, o risco e o ambiente institucional (FAGUNDES, 1998). Portanto, uma vez que se percebe a existência de custos que muitas vezes são negligenciados pelas teorias econômicas, fica nítida a importância de estudar e analisar as transações tendo como propósito melhorar a sua eficiência, de modo a minimizar os custos que, de certo modo, podem ser implícitos.

O estudo da teoria dos custos de transação, normalmente, possui um enfoque dentro da teoria da firma, entretanto ele pode ser estendido a diversos outros campos da teoria econômica. Este artigo diferencia-se por oferecer uma visão dessa teoria dentro da linha de finanças, mais especificamente, dentro do campo de estudos referentes ao mercado de capitais. Portanto, neste caso, a palavra transação se refere à negociação de ativos financeiros em bolsa de valores. Percebe-se que, nessa interação, incidem custos de transação que podem ser caracterizados como: (1) custos explícitos, que são de fácil percepção, como as despesas referentes às taxas de corretagem, às taxas de emolumentos, às taxas de liquidação e aos impostos; (2) custos implícitos, que não são diretamente observados, como a assimetria de informações entre os compradores e os vendedores (SANVICENTE, 2011), o impacto do mercado e os custos de oportunidade (ANTONIAZZI, 2013).

Dentro do contexto dos custos implícitos inerentes ao mercado acionário, pode-se acrescentar o que se denomina risco de liquidez. Define-se, nesse ponto, um ativo como líquido se ele puder ser comprado ou vendido rapidamente ao preço corrente de mercado e a um baixo custo (AMIHUD; MEDELSON, 1988). Portanto, percebe-se que a inexistência de liquidez de um ativo financeiro no mercado acionário pode ser identificada como um fator de risco para os investidores (MAJEWSKI et al., 2014), uma vez que um ativo financeiro ilíquido pode exigir mais tempo para ser comercializado e, se houver, por exemplo, um grande volume de negociação na ponta vendedora, o ativo poderá incorrer em uma queda acentuada em seu preço (ANTONIAZZI, 2013).

Portanto, a iliquidez de um ativo financeiro representa um custo de transação. Esse custo implícito pode ser amenizado pelo que se denomina de prêmio de liquidez, que se refere a uma compensação na taxa de retorno proporcionada pelo risco assumido. Observa-se que ativos com baixa liquidez devem possuir uma taxa de retorno mais elevada do que ativos com alta liquidez, pois o investidor que renunciar a liquidez, comprando um ativo ilíquido, estará assumindo um risco maior devido aos custos de transação implícitos inerentes à negociação e, portanto, ao assumir um investimento com esse perfil, deve ser recompensado por um prêmio. Esse prêmio é denominado de prêmio de liquidez (MACHADO; MEDEIROS, 2012).

Estudos referentes ao trade-off entre liquidez e retorno no mercado financeiro são relativamente recentes. As primeiras pesquisas sobre o tema foram realizadas por Amihud e Mendelson, no ano de 1986, nas quais propuseram uma correlação positiva entre iliquidez e retorno. No Brasil, ainda existe uma lacuna 
a ser preenchida a respeito de custos de transação que envolve as negociações no mercado de capitais, principalmente referentes aos custos relacionados ao trade-off entre liquidez e retorno.

Desse modo, emerge o seguinte problema de pesquisa: Há indicativos de que os investidores recebem um retorno adicional devido aos custos de transação provocados por reduções na liquidez? Tendo como objetivo verificar a existência, dentro do mercado acionário brasileiro e americano, de um prêmio pelo risco assumido pelos investidores referente ao custo de transação inerente à falta de liquidez na negociação de ativos financeiros. O propósito de incluir o mercado acionário americano neste estudo é o de realizar uma comparação entre o mercado de capitais de um país desenvolvido com o mercado de capitais de um país em desenvolvimento, a respeito da existência de um prêmio de liquidez.

Para que tal objetivo seja alcançado, este trabalho utilizará uma adaptação de um índice, denominado índice Beta, que mede o grau em que o retorno de um ativo financeiro está correlacionado com o mercado acionário. Portanto, esse índice permitirá observar a existência de um prêmio de liquidez condicional, que fornece uma medida em que se verifica se os ativos com pouca liquidez e, consequentemente, com maior custo de transação, fornecem uma compensação na sua taxa de retorno, uma vez que esses investimentos apresentam um risco superior de negociação do que os investimentos em ativos líquidos. Para que o índice Beta seja calculado, é necessária a estimação das variâncias e das covariâncias das variáveis utilizadas. Portanto, para obter essas estimativas, este artigo utiliza-se do procedimento estatístico denominado Cópula-DCC-GARCH. Esse procedimento, assim como o índice Beta, será discutido com mais detalhes na seção de metodologia.

Assim, este estudo busca contribuir para a literatura existente em três direções importantes. Primeiramente, através do relacionamento de uma teoria, inicialmente voltada para a teoria da firma, com as teorias da linha de pesquisa de finanças, referentes ao mercado de capitais. Segundo, com a comparação da existência de um prêmio referente ao risco assumido pelo investidor pela falta de liquidez entre um mercado acionário de um país desenvolvido e um mercado acionário de um país em desenvolvimento. Terceiro, através da utilização de um método inovador para a estimação do índice Beta para liquidez, que permite verificar a relação entre liquidez e retorno ao longo do tempo.

\section{Custos de Transação e a Liquidez Acionária}

A literatura não apresenta um conceito preciso de liquidez acionária. Liquidez, geralmente, é definida como a capacidade de se negociar rapidamente uma grande quantidade de um ativo sem que seu preço apresente variações substanciais (LIU, 2006). Termos como "rapidamente", "grande" e "substanciais" tendem a ser subjetivos, o que torna difícil o estabelecimento de uma medida única e correta de liquidez.

A liquidez, por definição, está intimamente relacionada aos custos de transação. O movimento de preço após uma transação é um dos componentes dos custos de transação, denominado impacto de mercado. Custos de transação são aqueles incorridos pelo investidor quando da execução de uma estratégia de investimento ou liquidação de uma carteira. Envolve todos os custos percebidos e não percebidos que afetam o resultado do negócio realizado (GASTINEAU; KRITZMAN, 1996). Os custos de transação envolvem, além da taxa de corretagem paga para a realização do negócio, outros custos, que eventualmente são até mais importantes, mas que permanecem implícitos e, diversas vezes, imperceptíveis para os agentes.

O custo de transação de ativos apresenta três componentes: primeiro, o spread de compra e venda, que é o diferencial de preço entre a oferta de compra e a de venda cotada; segundo, o custo de oportunidade, associado ao tempo para a execução de uma determinada ordem, pois a espera pela realização de um negócio pode fazer com que o mesmo deixe de ser lucrativo ou vantajoso; e, terceiro, o impacto no preço, que, dentre todos os custos de transação, é o mais impacta na liquidez; Gastineau e Kritzman (1996) afirmam que representa a maior parte do custo de transação em uma operação de grande porte, sendo definido como a mudança do preço de um determinado ativo em função de uma transação realizada. 
Ativos menos líquidos demandam uma taxa de retorno maior que ativos mais líquidos, uma vez que para abrir mão da liquidez e assumir custos maiores em negociações futuras, em razão da baixa liquidez da ação, os investidores, provavelmente, exigiriam um prêmio para assumir investimentos com esse perfil. Logo, os preços de ativos não líquidos precisam cair suficientemente para atrair investidores. Segundo Amihud e Mendelson (1988, 1991, 2006), um ativo é líquido se ele puder ser comprado ou vendido rapidamente ao preço corrente de mercado e a um baixo custo. Portanto, a liquidez pode estar relacionada ao custo de realizar uma transação no mercado de capitais.

A teoria de precificação de ativos sugere que o retorno esperado de um ativo seja crescente com seu nível de risco, porque investidores avessos ao risco requerem uma compensação para aceitar mais risco. Uma vez que os investidores também são avessos ao custo de iliquidez e querem ser recompensados para enfrentá-lo, o retorno esperado de um ativo é função crescente da iliquidez. Assim, o retorno de um ativo depende de duas características: risco e liquidez (AMIHUD; MENDELSON, 2006). Para Jacoby, Fowler e Gottesman (2000), risco e liquidez são variáveis inseparáveis. Logo, ao avaliar ativos, os analistas financeiros devem levar em consideração não somente o risco e retorno esperado do ativo, mas também sua liquidez.

Minardi, Sanvicente e Monteiro (2005) investigaram o mercado brasileiro no período compreendido entre 1998 a 2003. Os autores analisaram a relação do bid-ask spread relativo (ou simplesmente spread, sendo o bid-ask spread em reais dividido pela média entre a última melhor cotação de compra e a última melhor cotação de venda), com o retorno das ações e com a assimetria de informações. Foram realizadas regressões aparentemente não relacionadas com os dados em painel. Quanto aos ativos individuais, os resultados mostraram uma relação negativa e significante entre o retorno das ações e iliquidez, sugerindo um prêmio de liquidez negativo. Já em relação às carteiras de ativos, na tentativa de validar o resultado anterior, a relação apresentou-se negativa, porém não significativa. A existência de prêmio por liquidez negativo no Brasil, apesar de não validado, se mostrou um resultado contrário ao encontrado no mercado americano e ao que seria esperado. Também observaram que o custo de transações é menor à medida que aumenta a transparência das informações. Amihud e Mendelson (1986) também acessaram o fato de que investidores demandam um prêmio por ativos menos líquidos, ceteris paribus, a fim de compensar custos de transação à época da negociação dos ativos.

Machado e Medeiros (2012) analisaram se existe o efeito liquidez no mercado acionário Brasileiro. Para isso, buscaram as empresas com ações negociadas na Bovespa no período de 1995 a 2008. Identificaram não só que existe um prêmio de liquidez no mercado brasileiro, mas também que uma empresa pode aumentar seu valor de mercado, melhorando a liquidez dos seus títulos e ações, uma vez que quanto menor o custo de capital, maior o valor da empresa.

Amihud e Mendelson (1986) desenvolveram um modelo que mostra como a liquidez afeta os preços dos ativos. O modelo caracteriza ativo por seus custos de transação e investidores por seu horizonte de investimento. O modelo foca na iliquidez e, nele, os investidores possuem planos heterogêneos de manutenção do investimento, ou seja, liquidação. Os investidores racionais selecionam ativos que maximizam seus retornos esperados, líquidos dos custos de transação e, em equilíbrio, ativos com maiores spreads são alocados aos investidores que mantêm planos de investimento com maior prazo de maturação (efeito clientela). Como resultado, a relação entre iliquidez e retorno é crescente e côncava, isto é, é menos crescente para ativos menos líquidos, que são mantidos por investidores de longo prazo, que podem depreciar seus custos de transação ao longo do período.

Enquanto os custos de liquidez de uma simples transação são baixos, relativos ao preço dos ativos, seu efeito cumulativo é maior, porque eles são incorridos repetidamente ao longo do tempo. Assim, a reação dos custos de iliquidez deveria ser, no mínimo, igual ao valor presente de todos os custos incididos no tempo. Por isso, investidores evitam investimentos em títulos ilíquidos caso não sejam adequadamente compensados. Em contrapartida, o preço de ativos ilíquidos precisa diminuir suficientemente para atrair investidores (AMIHUD; MENDELSON, 1988, 1991, 2006).

Um investidor pode reduzir o risco de manter um título formando uma carteira diversificada, porém não há nada que ele possa fazer para evitar os custos de iliquidez. Ele vai incorrer nesses custos sempre 
que comprar ou vender um título, e manter uma carteira de ativos ilíquidos não irá eliminar os custos de transação, que são sempre adicionais (AMIHUD; MENDELSON, 1989). Isto significa que uma carteira de ativos ilíquidos permanece ilíquida.

De acordo Amihud e Mendelson (1988, 1991, 2006), iliquidez pode ser gerenciada, pois se pode estabelecer políticas corporativas que aliviem os custos de iliquidez aos investidores, ou seja, desenvolver políticas que melhorem a liquidez dos títulos, pois, para uma empresa que emite títulos ou ações, quanto menor o retorno requerido, menor o seu custo de capital. Tanto que uma empresa pode aumentar seu valor de mercado, melhorando a liquidez dos seus títulos e ações, pois quanto menor o custo de capital, maior o valor da empresa.

Amihud e Mendelson $(1988,1991,2006)$ estabelecem estratégias que as empresas possam empregar para aumentar a liquidez de seus títulos, dentre as quais, destacam-se: aumentar a base de investidores da empresa, especialmente atraindo pequenos investidores; prover voluntariamente mais informações ao mercado, reduzindo a assimetria de informações; diminuir a emissão de títulos e ações fragmentadas e listar a empresa em bolsa de valores mais líquidas.

A teoria de precificação de ativos sugere que o retorno esperado de um ativo seja crescente com seu nível de risco, porque investidores avessos ao risco requerem uma compensação para aceitar mais risco. Uma vez que os investidores também são avessos ao custo de iliquidez e querem ser recompensados para enfrentá-lo, o retorno esperado de um ativo é função crescente da iliquidez (FAMA; FRENCH, 2004).

\section{Materiais e Métodos}

Amplamente utilizado em finanças, o Beta de Mercado $(\beta)$ mede o grau em que o retorno de um ativo está correlacionado com o mercado em geral. Ele representa o risco sistemático, que não pode ser eliminado através de carteiras de diversificações e, apesar de extensas criticas, ainda é amplamente utilizado em estratégias financeiras. No contexto do tradicional modelo de precificação de ativos de capital (CAPM), introduzido por Sharpe (1964) e Lintner (1965), o $\beta$ é assumido como sendo invariante no tempo e é, então, estimado através de Mínimos Quadrados Ordinários (MQO). No entanto, o pressuposto da estabilidade desse coeficiente tem sido questionado em muitos estudos, como Sunder (1980), Kim (1993), Altinsoy (2009) e Zhou (2013).

Nesse sentido, para atingir o objetivo anteriormente proposto, este trabalho empregará o conceito por trás do Índice Beta para verificar a existência de um prêmio por liquidez condicional e. assim. averiguar se os acionistas que investem em ativos com menor liquidez, e que consequentemente apresentam um maior custo de transação, recebem uma compensação por esse investimento de risco superior. Para realização deste objetivo, serão utilizados dados diários das cotações do Índice Bovespa e do Índice Dow Jones Industrial Average, bem como seus respectivos volumes de negociação em dólares americanos como proxys de liquidez. A amostra escolhida, por sua vez, corresponde ao período de 03/01/2000 a 27/04/2015, compreendendo mais de 15 anos de negociações do mercado acionário americano e brasileiro, perfazendo um total de 3686 observações diárias.

Tsay (2010) destaca que, na literatura financeira, séries de retornos de ativos são comumente tratadas como estacionárias. Nesse contexto, a fim de evitar problemas de nãoestacionariedade, além de padronizar a análise, optou-se por empregar os log-retornos das variáveis utilizadas. O calculo de um Índice Beta exige a estimação das covariâncias e variâncias dos respectivos pares de variáveis. O procedimento estatístico escolhido para obter essas estimativas foi o modelo Copula-DCC-GARCH, que é discutido por Jondeau e Rockinger (2006).

O emprego do modelo Copula-DCC-GARCH é esquematicamente exposto por Righi e Ceretta (2012), e será apresentado neste artigo como descrito pelos autores. O primeiro passo consiste em empregar um modelo de vetor auto-regressivo (VAR), a fim de obter a estimativa média do retorno e da série de resíduos de cada variável. A equação (1) apresenta a formulação matemática do modelo VAR utilizado:

$$
\boldsymbol{r}_{t}=\boldsymbol{\phi}_{0}+\boldsymbol{\Phi}_{1} \boldsymbol{r}_{t-1}+\cdots \boldsymbol{\Phi}_{p} \boldsymbol{r}_{t-p}+\boldsymbol{a}_{t}
$$


Em (1), $r_{t}$ é um vetor $k$-dimensional dos log-retornos no período $t$; $\phi_{0}$ é um vetor $k$-dimensional de constantes; $\phi_{1}, i=1, \ldots, p$ são matrizes $k \times k$ de parâmetros; $\left\{a_{t}\right\}$ é uma sequência de vetores aleatórios serialmente não correlacionados com média zero e matriz de covariância $\Sigma_{\mathrm{a}}$. Posteriormente, utilizando os resíduos obtidos através do modelo VAR, foi estimada a matriz de covariância condicional com o modelo Copula-DCC-GARCH, representado pela formulação (2):

$$
\boldsymbol{r}_{t}=\boldsymbol{\phi}_{0}+\boldsymbol{\Phi}_{1} \boldsymbol{r}_{t-1}+\cdots \boldsymbol{\Phi}_{p} \boldsymbol{r}_{t-p}+\boldsymbol{a}_{t}
$$

Onde,

$$
\begin{aligned}
& \boldsymbol{\Omega}_{t}=\boldsymbol{D}_{t}{ }^{\prime} \boldsymbol{R}_{t} \boldsymbol{D}_{t} . \\
& \boldsymbol{D}_{t}=\operatorname{diag}\left(h_{1, t}^{1 / 2} \ldots h_{N, t}^{1 / 2}\right), h_{i, t}^{2}=c_{i}+\sum_{p} b_{i, p} h_{i, t-p}^{2}+\sum_{q} a_{i, q} \varepsilon_{i, t-q}^{2} ; \\
& \boldsymbol{R}_{t}=\operatorname{diag}\left(q_{11, t}^{-1 / 2} \ldots q_{N N, t}^{-1 / 2}\right) \boldsymbol{Q}_{t} \operatorname{diag}\left(q_{11, t}^{-1 / 2} \ldots q_{N N, t}^{-1 / 2}\right) ; \\
& \boldsymbol{Q}_{t}=(1-\alpha-\beta) \bar{Q}+\alpha u_{t-1} u_{t-1}^{\prime}+\beta \boldsymbol{Q}_{t-1}, u_{i, t}=\varepsilon_{i, t} / \sqrt{h_{i, t}}
\end{aligned}
$$

$\bar{Q}$ é a matriz $N \times N$ composta pela covariância incondicional de $u_{i, t}$; $\alpha$ e $\beta$ são parâmetros não negativos, satisfazendo $\alpha+\beta<1$. Os parâmetros foram estimados conforme o procedimento quase máxima verossimilhança em duas etapas, conforme Jondeau et al. (2007). No primeiro estágio, a variância condicional é estimada através de um modelo GARCH univariado para cada ativo. Em seguida, com base nos parâmetros do primeiro estágio são estimados os parâmetros para a covariância condicional.

Para definir as distribuições marginais das inovações e conjunta (Cópula), foi empregue o Critério de Informação de Akaike (AIC). As distribuições candidatas foram a Normal, $t$ de Student, bem como suas versões assimétricas para o primeiro estágio, além de Copula Multivariada Normal e Copula Multivariada $t$ de Student para o segundo. De acordo com Bouyé (2000), cópulas só foram empregadas em finanças a partir de 1999 e podem ser entendidas como distribuições de probabilidade multivariadas, em que a distribuição marginal de probabilidade de cada variável é uniforme. Mais de detalhes sobre o assunto podem ser vistos em Jondeau e Rockinger (2006) e Righi e Ceretta (2012).

Para validar o modelo, buscou-se identificar a presença de correlação serial nos resíduos, nos resíduos quadrados do modelo VAR e nos resíduos quadrados do modelo Copula-DCC-GARCH, por meio do teste $Q$ de Ljung e Box (1978), representado pela formulação (6). Essa estatística testa a hipótese nula de que os dados são aleatórios contra a alternativa de não aleatoriedade dos mesmos.

$$
Q=n(n+2) \sum_{k=1}^{h} \frac{\widehat{\rho}_{k}^{2}}{n-k}
$$

Na formulação (3), $n$ é o tamanho da amostra; $\hat{\rho}_{k}^{2}$ é a autocorrelação da amostra na defasagem $k$; $h$ é o número de defasagens sendo testadas. A estatística $Q$, de Ljung e Box, segue a distribuição de quiquadrado $\left(\chi^{2}\right)$, com $h$ graus de liberdade.

Após a estimação das matrizes de covariâncias pelo modelo $\mathrm{GARCH}$, a relação entre retorno e liquidez será calculada através do mesmo procedimento utilizado por Zhou (2013) para obter o Beta condicional de mercado. Desse modo, representando a associação entre a liquidez e o retorno dos ativos. A equação (4) apresenta a formulação do Beta condicional para a liquidez:

$$
\left.\widehat{\beta}_{t}=\frac{\operatorname{Cov}\left(R_{t}, L_{t}\right)}{\operatorname{Var}\left(L_{t}\right)}\right)
$$

Na formulação (4), é o retorno do índice acionário ao longo de $t$; $L_{t}$ é o volume do respectivo índice em $t$; Cov e Var são as covariâncias e variâncias condicionais estimadas pelo modelo Copula-DCC-GARCH. 


\section{Análise dos Resultados}

Inicialmente, com o intuito de evidenciar a evolução das séries estudadas, foram apresentados gráficos das variáveis em nível e em primeira diferença de logaritmos (log-retornos). A Figura 1 expõe o comportamento temporal destas variáveis.

Figura 1 - Evolução temporal do Índice Bovespa, do volume negociado pelo Índice Bovespa, do Índice Dow Jones, e do volume negociado pelo índice Dow Jones e seus respectivos log-retornos para o período de janeiro de 2000 a abril de 2015.
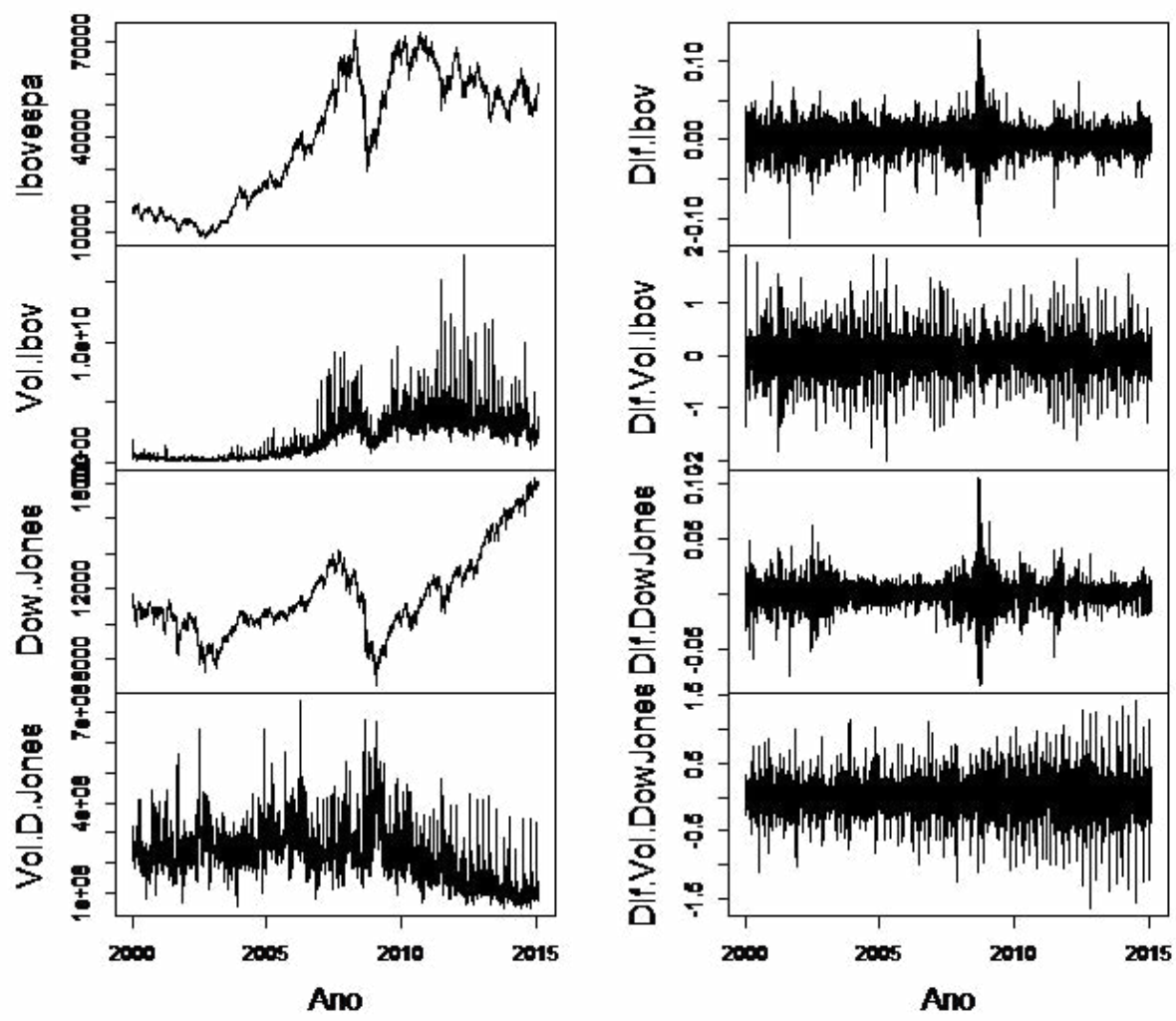

Fonte: Elaborado pelo autor.

Observou-se. na Figura 01. que tanto o Ibovespa como o Dow Jones apresentaram uma tendência de crescimento até 2008 , que foi interrompida pela crise do sub-prime, que se intensificou no período. Durante a crise, ambos os índices exibiram significativas reduções em seus valores. Porém, ainda no mesmo ano, o lbovespa demonstrou uma recuperação até meados de 2009, mantendo um desenvolvimento estável no restante do período, e o Dow Jones apresentou um crescimento constante que se manteve até o final da amostra. O volume do Ibovespa, por sua vez, evoluiu de forma constante ao longo do período, o que sugere uma maior consolidação do mercado brasileiro. Já o do Dow Jones apresentou uma tendência de redução em seu volume negociado a partir de 2010, que se manteve até final do período estudado.

Na segunda parte da Figura 1 é exposta a evolução temporal das variáveis em log-retorno, tais como utilizadas na estimação dos modelos. De um modo geral, todas possuem um comportamento típico de séries estacionárias, o que é esperado, uma vez que a transformação em log-retorno é o procedimento padrão empregado na literatura para obtenção de séries financeiras estacionárias, o que permite, além de evitar problemas de inferência estatística, padronizar os resultados obtidos.

Dando continuidade a análise, na Tabela 1 são apresentadas as estatísticas descritivas das séries em nível da Figura 1. Os resultados evidenciaram que estas apresentam médias e desvios padrões de diferentes magnitudes, o que se deve à utilização de variáveis com formação distinta e reitera a necessidade do emprego dos log-retornos a fim de padronizar as informações. Os pontos de mínimo e máximo, em geral, apresentam valores com grande dispersão entre si, e que não se refletem inteiramente em seus desvios padrões, indicando uma significativa variação na série durante os mais de 15 anos estudados. 
Tabela 1 - Estatísticas descritivas do Índice Bovespa e do Índice Dow Jones e seus respectivos volumes. negociados

\begin{tabular}{|l|r|r|r|r|}
\hline Variável & Ibovespa & Vol. Ibovespa & Dow Jones & Vol. Dow Jones \\
\hline Média & $40.162,8000$ & $1.985 .427 .000,0000$ & $11.601,2245$ & $218.394 .100,0000$ \\
\hline D. Padrão & $19.986,8700$ & $1.713 .661 .000,0000$ & $2.408,3012$ & $90.110 .190,0000$ \\
\hline Mínimo & $8.370,0000$ & $58.991 .930,0000$ & $6.547,0500$ & $47.230 .560,0000$ \\
\hline Máximo & $73.516,0000$ & $17.367 .090 .000,0000$ & $18.288,6300$ & $738.442 .400,0000$ \\
\hline Assimetria & $-0,1382$ & 1,2805 & 0,8846 & 0,7754 \\
\hline Curtose & $-1,4838$ & 4,4424 & 0,3091 & 1,6894 \\
\hline
\end{tabular}

Fonte: Elaborado pelo autor.

Ademais, com exceção do lbovespa, as variáveis são positivamente assimétricas, havendo uma maior frequência de observações à esquerda da média. Além disso, ambos os índices e o volume negociado pelo Dow Jones apresentaram uma distribuição platicúrtica com menor incidência de valores extremos. Por outro lado, o volume do Ibovespa se mostrou leptocúrtico, com uma frequência mais concentrada e uma maior presença de valores extremos. Isto, de modo geral, indica que nenhuma variável apresenta um comportamento probabilístico característico de séries normalmente distribuídas.

Após essa breve descrição dos dados, a próxima etapa do estudo consistiu na estimação de um modelo VAR como descrito pela Equação (1). Foram estimados dois modelos, um para os log-retornos do Ibovespa e outro para o Dow Jones, cada qual com seus respectivos volumes negociados. Para a escolha das defasagens, por sua vez, empregou-se o critério de informação AIC. De todo modo, por não ser o escopo deste artigo, os resultados das estimações do modelo VAR não serão apresentados. A fim de validar o modelo acima, buscou-se identificar a presença de correlação serial nos resíduos e resíduos ao quadrado, por meio do teste $Q$ de Ljung e Box (1978). Os resultados do teste $Q$ são apresentados na Tabela 2.

Tabela 2 - Teste $Q$ dos resíduos em nível e quadráticos para os modelos VAR estimados para os índices Bovespa e Dow Jones e com seus respectivos volumes.

\begin{tabular}{lcccc}
\hline Resíduos & $\begin{array}{c}\text { Retorno } \\
\text { (nível) }\end{array}$ & $\begin{array}{c}\text { Volume } \\
\text { (nível) }\end{array}$ & $\begin{array}{c}\text { Retorno } \\
\text { (quadrático) }\end{array}$ & $\begin{array}{c}\text { Volume } \\
\text { (quadrático) }\end{array}$ \\
\hline Dow Jones & & & & \\
Estatística (1 lag) & $<0,0001$ & 0,0005 & 65,2911 & 41,0699 \\
P-valor & $(0,9943)$ & $(0,9814)$ & $(<0,0001)$ & $(<0,0001)$ \\
Estatística (2 lags) & 0,0001 & 0,0032 & 505,1791 & 41,1752 \\
P-valor & $(0,9999)$ & $(0,9983)$ & $(<0,0001)$ & $(<0,0001)$ \\
Estatística (3 lags) & 0,0040 & 0,0090 & 635,3136 & 41,2303 \\
P-valor & $(0,9999)$ & $(0,9997)$ & $(<0,0001)$ & $(<0,0001)$ \\
Estatística (4 lags) & 0,0063 & 0,0142 & 866,1788 & 41,2513 \\
P-valor & $(0,9999)$ & $(0,9999)$ & $(<0,0001)$ & $(<0,0001)$ \\
Estatística (5 lags) & 0,0064 & 0,0153 & 1099,4892 & 45,2076 \\
P-valor & $(0,9999)$ & $(0,9999)$ & $(<0,0001)$ & $(<0,0001)$ \\
\hline Ibovespa & & & & \\
Estatística (1 lag) & 0,0026 & 0,0098 & 85,4594 & 11,3089 \\
P-valor & $(0,9586)$ & $(0,9210)$ & $(<0,0001)$ & $(0,0007)$ \\
Estatística (2 lags) & 0,0032 & 0,0140 & 410,3923 & 11,9992 \\
P-valor & $(0,9983)$ & $(0,9929)$ & $(<0,0001)$ & $(0,0024)$ \\
Estatística (3 lags) & 0,0060 & 0,0155 & 535,6421 & 15,7652 \\
P-valor & $(0,9998)$ & $(0,9994)$ & $(<0,0001)$ & $(0,0012)$ \\
Estatística (4 lags) & 0,0061 & 0,0267 & 670,6129 & 17,7916 \\
P-valor & $(0,9999)$ & $(0,9999)$ & $(<0,0001)$ & $(0,0013)$ \\
Estatística (5 lags) & 0,0080 & 0,0300 & 841,7920 & 19,6315 \\
P-valor & $(0,9999)$ & $(0,9999)$ & $(<0,0001)$ & $(0,0014)$ \\
\hline
\end{tabular}

Fonte: Elaborado pelo autor. 
Na Tabela 2 são apresentados os resultados do teste $Q$, em que foram testadas 5 defasagens e não houve valor significativo para o teste $Q$ dos resíduos do modelo. No entanto, quando consideradas suas formas quadráticas, as defasagens se mostraram estatisticamente significativas. Esse resultado confirma a presença do efeito heteroscedástico condicional, sugerindo que a volatilidade dos resíduos pode ser modelada. Desse modo, utilizando os resíduos obtidos através do modelo VAR, foi estimada uma matriz de covariância condicional com um modelo Cópula-DCC-GARCH para cada índice com seu respectivo volume. A formulação matemática do modelo foi apresentada na Equação (2) e os resultados são apresentados na Tabela 3.

Tabela 3 - Modelo Cópula-DCC-GARCH estimado para os índices Bovespa e Dow Jones e seus respectivos volumes

\begin{tabular}{ccccc}
\hline & \multicolumn{2}{c}{ Dow Jones } & & \multicolumn{2}{c}{ Volume Dow Jones } \\
Coeficiente & Estatística & p-valor & Estatística & p-valor. \\
\hline 0,0000 & 1,0000 & 0,0000 & 1,0000 \\
0,0000 & 0,9790 & 0,0000 & 0,5501 \\
0,0500 & 0,0201 & 0,0550 & $<0,0001$ \\
Shape & 0,9000 & $<0,0001$ & 0,9017 & $<0,0001$ \\
& 4,0003 & $<0,0001$ & 4,1023 & $<0,0001$ \\
\hline Coeficiente & 0,0144 & 0,0723 & & \\
& 0,9389 & $<0,0001$ & & V-valor \\
\hline & 10,5929 & $<0,0001$ & & 1,0000 \\
& Ibovespa & & 0,5834 \\
Estatística & $\mathbf{p}$-valor & Estatística & $<0,0001$ \\
& 0,0000 & 1,0000 & 0,0000 & $<0,0001$ \\
& 0,0000 & 0,3370 & 0,0000 & $<0,0001$ \\
\hline 0,0500 & $<0,0001$ & 0,0523 & \\
& 0,9000 & $<0,0001$ & 0,9008 & \\
\hline & 4,0009 & $<0,0001$ & 4,0405 & \\
\hline
\end{tabular}

Fonte: Elaborado pelo autor.

Na Tabela 3, os parâmetros $\mu$ confirmam o fato do modelo VAR anteriormente estimado ter captado as dependências seriais das séries temporais utilizadas, o que também pode ser observado pela não significância das respectivas estatísticas $Q$ na Tabela 2 . Adicionalmente, todas as volatilidades se mostraram afetadas pela sua volatilidade passada ao nível de $5 \%$ de significância. Essa característica é evidenciada pela significância estatística do parâmetro . Além disso, a volatilidade também demonstrou ser influenciada pelos choques quadrados não antecipados em suas taxas de retorno, como pode ser notado pela significância estatística do parâmetro . Como outro aspecto relevante, destaca-se que a cópula $t$ de Student apresentou significância estatística na especificação conjunta dos dados, o que pode ser observado no parâmetro Joint mshape para ambos os modelos.

Para verificar o ajuste do modelo Cópula-DCC-GARCH, do mesmo modo que para o modelo VAR, buscou-se identificar a presença de correlação serial nos seus resíduos ao quadrado, por meio do teste $Q$ de Ljung e Box (1978). Os resultados do teste $Q$ são apresentados na Tabela 4. 
Tabela 4 - Teste $Q$ dos resíduos quadráticos dos modelos Cópula-DCC-GARCH estimados para os índices Bovespa e Dow Jones e seus respectivos volumes.

\begin{tabular}{lcccc}
\hline Resíduos Quadráticos & $\begin{array}{c}\text { Retorno } \\
\text { (Dow Jones) }\end{array}$ & $\begin{array}{c}\text { Volume } \\
\text { (Dow Jones) }\end{array}$ & $\begin{array}{c}\text { Retorno } \\
\text { (Ibovespa) }\end{array}$ & $\begin{array}{c}\text { Volume } \\
\text { (Ibovespa) }\end{array}$ \\
\hline Estatística (1 lag) & 3,7128 & 0,0011 & 1,8599 & 0,8640 \\
P-valor & $(0,0539)$ & $(0,9730)$ & $(0,1726)$ & $(0,3525)$ \\
Estatística (2 lags) & 4,9212 & 1,2447 & 2,1371 & 1,0059 \\
P-valor & $(0,0853)$ & $(0,5366)$ & $(0,3435)$ & $(0,6047)$ \\
Estatística (3 lags) & 5,3268 & 4,1562 & 3,3851 & 1,1717 \\
P-valor & $(0,1493)$ & $(0,2450)$ & $(0,3359)$ & $(0,7597)$ \\
Estatística (4 lags) & 5,5103 & 5,9791 & 4,1051 & 4,8856 \\
P-valor & $(0,2388)$ & $(0,2007)$ & $(0,3919)$ & $(0,2992)$ \\
Estatística (5 lags) & 6,3721 & 6,0199 & 4,9625 & 8,9767 \\
P-valor & $(0,2716)$ & $(0,3042)$ & $(0,4204)$ & $(0,1099)$ \\
\hline
\end{tabular}

Fonte: Elaborado pelo autor.

Na Tabela 4 são apresentados os resultados do teste $Q$ para os resíduos quadráticos de ambos os modelos estimados. Novamente foram testadas 5 defasagens e não houve valor significativo para o teste $Q$ dos resíduos quadráticos dos modelos, o que indica a aleatoriedade dos resíduos quanto à sua dependência com o passado, e sugere que a informação contida nos dados foi captada pelo modelo.

Após a estimação das matrizes de covariâncias pelo modelo Copula-DCC-GARCH, o Beta condicional para liquidez foi calculado como descrito pela Equação (4). A relação entre retorno e liquidez estimada para o mercado acionário americano é apresentado na Figura 2.

Figura 2 - Evolução temporal do Beta condicional de liquidez para o mercado acionário americano estimado pelo relacionamento entre o Índice Dow Jones e seu volume negociado.

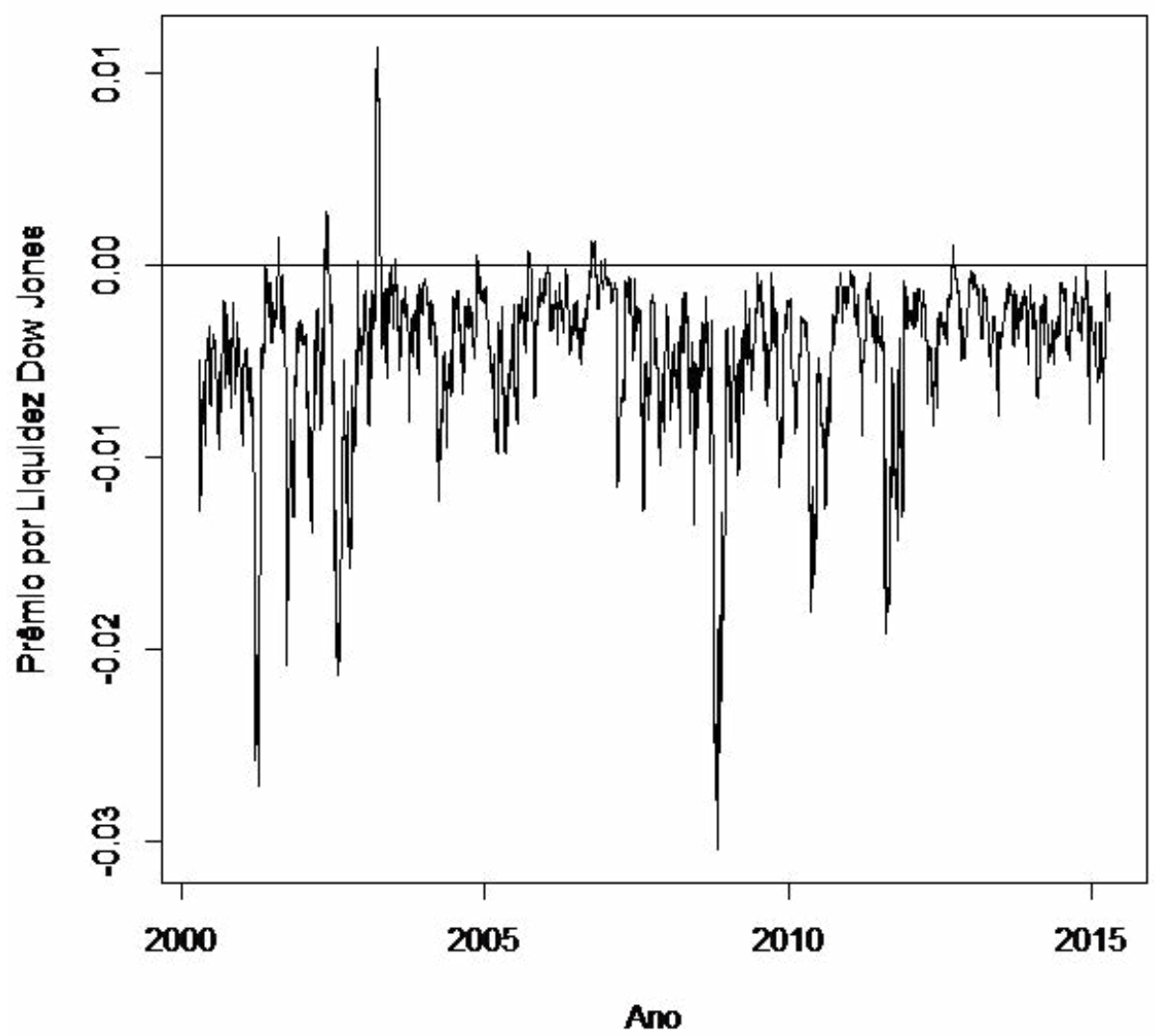

Fonte: Elaborado pelo autor. 
Na Figura 2 é exposto o Beta condicional de liquidez para o Índice Dow Jones. Os valores observados na figura podem ser analisados de forma similar aos coeficientes de uma regressão de Mínimos Quadrados Ordinários, ou seja, se os valores do volume variarem em uma unidade os valores do retorno estarão variando em $x$ unidades. Todas as variáveis empregadas foram padronizadas para log-retornos, desse modo os valores observados estão em unidades percentuais, isto é, quando o volume negociado pelo Dow Jones varia em uma unidade percentual o retorno do índice se move $x$ unidades percentuais, com esse valor mudando a cada período $t$.

Assim, na Figura 2 percebe-se que o volume e o retorno do Índice Dow Jones apresentaram um relacionamento negativo em quase todos os períodos dos mais de 15 anos estudados. Tal relacionamento indica que, quando o volume negociado aumenta, o retorno das ações diminui; e, de forma similar, quando o volume negociado diminui, o retorno das ações aumenta. Essa associação sugere a existência de um prêmio por liquidez no mercado acionário norte americano, ou seja, os investidores que aplicam em ativos com uma menor liquidez recebem uma compensação (prêmio por liquidez) por empregar seus recursos em ações que possuem um maior custo de transação por serem menos líquidas. Além disso, destaca-se, na Figura 2, que o relacionamento das variáveis não é constante ao longo do tempo, com o momento de maior oscilação ocorrendo durante 2008 , o que coincide com a crise do sub-prime que afetou os mercados financeiros de todo o mundo.

Para Amihud e Mendelson (1991), esse efeito da liquidez pode ser comparado ao efeito risco sobre ativos de capital. Investidores avessos ao risco exigem maiores retornos esperados como compensação pelo risco maior. De forma similar, investidores preferem investir em ativos líquidos, que possam ser transacionados rapidamente e com baixo custo. Já que ativos pouco líquidos tendem a exigir um custo de transação maior quando da sua compra e venda, e, para que possam ser atrativos quando comparados a ativos mais líquidos, devem oferecer um retorno maior, para que os investidores se sintam atraídos em mantê-los em suas carteiras (VIEIRA; SILVEIRA; RIGHI, 2012).

Um grande número de estudos tem abordado a relação entre liquidez e retorno acionário para o mercado americano (AMIHUD; MENDELSON, 1988, 1991, 2006; DATAR et al., 1998; PASTOR; STAMBAUGH, 2003; LIU, 2006). No entanto, o mercado acionário brasileiro ainda apresenta um número reduzido de pesquisas sobre o tema, com estas, em sua maioria, buscando adicionar alguma proxy que represente a liquidez acionária ao modelo CAPM de precificação de ativos para verificar o impacto desta no retorno. Contudo, como observado na Figura 2, as variáveis apresentam um relacionamento que varia com o tempo e é sensível a crises, o que torna necessária a busca por novos métodos que captem de forma mais adequada esse relacionamento.

Nesse contexto, também foi estimado, da mesma forma que para o mercado americano, o relacionamento entre o retorno e o volume negociado pelo Índice Bovespa. Este relacionamento foi estimado como descrito pela Equação (4) é mostrado na Figura 3. 
Figura 3 - Evolução temporal do Beta condicional de liquidez para o mercado acionário brasileiro estimado pelo relacionamento entre o Índice Bovespa e seu volume negociado.

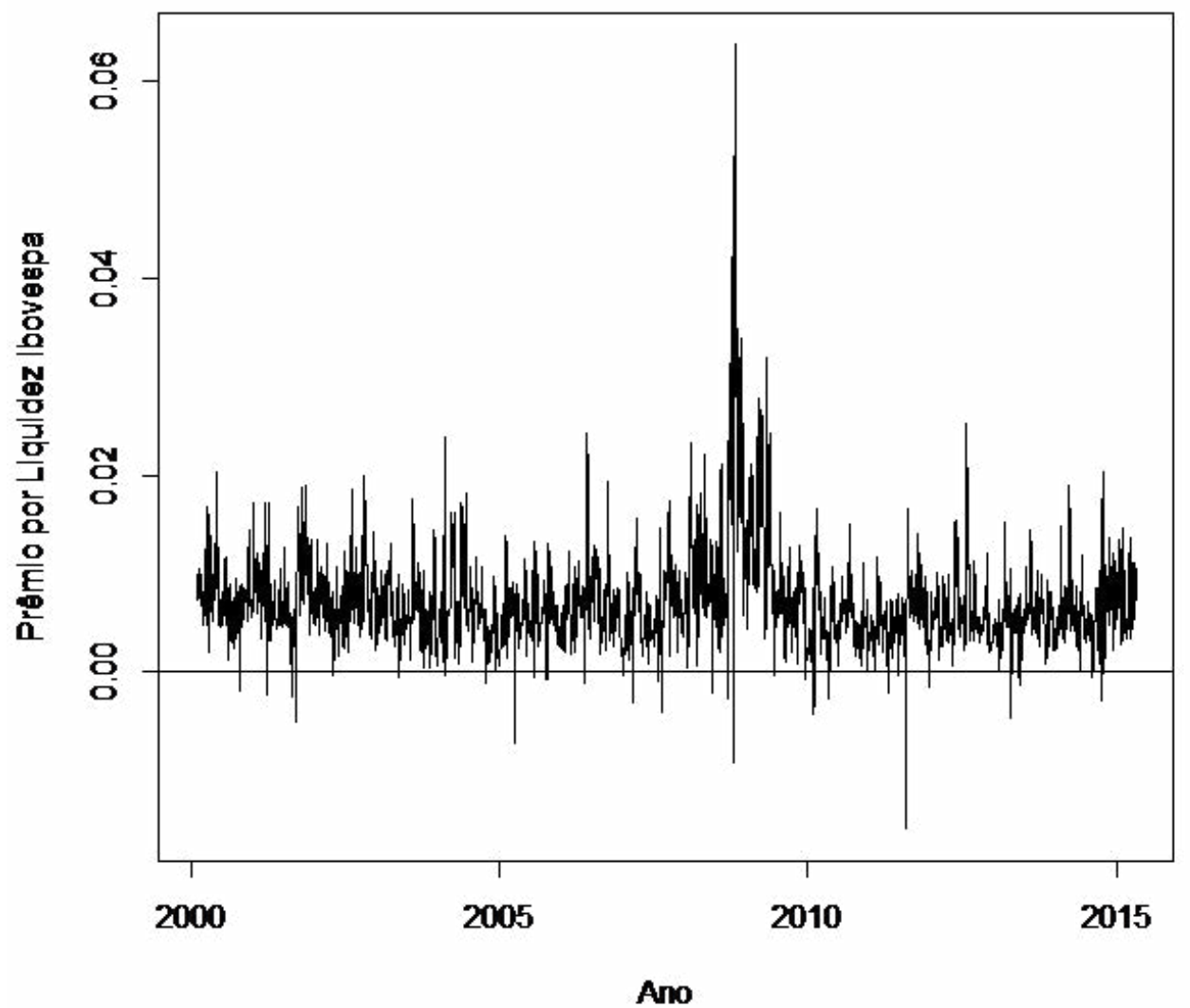

Fonte: Elaborado pelo autor.

Na Figura 3, o volume e o retorno acionário apresentaram um relacionamento positivo para quase todo o período estudado. Tal relacionamento indica que aumentos no volume negociado provocariam aumentos nos retorno dos ativos; e, de forma similar, as reduções no volume negociado provocariam reduções no retorno dos ativos. Essa associação se mostra o oposto do que foi observado para o mercado americano ao se utilizar variáveis que objetivam mensurar as mesmas características do mercado.

Esse resultado sugere que investidores que aplicam em ações com menor liquidez não recebem uma remuneração adicional por negociarem ativos que possuem um maior custo de transação. Por sua vez, investidores estariam recebendo uma remuneração adicional ao empregar seus recursos em ações mais líquidas, que possuem um menor custo de transação e, consequentemente, são menos arriscadas.

Esse resultado, se mostra contrário ao esperado, de acordo com a literatura norte-americana, como constatado por Datar et al. (1998) e Amihud (2002), uma vez que ativos com maior liquidez seriam menos arriscados e proporcionariam um menor retorno. No entanto, coincidem com os encontrados por Jun, Marathe e Shawky (2003) e Correia e Amaral (2012) para mercados emergentes, que sugerem que a liquidez pode estar funcionando como um fator de risco na precificação dos ativos e que esse fato tem ocorrido, principalmente, em mercados emergentes.

\section{Conclusão}

Este trabalho buscou verificar a existência, dentro do mercado acionário brasileiro e americano, de um prêmio pelo risco assumido pelos investidores referente ao custo de transação inerente à falta de liquidez na negociação de ativos financeiros. Como resultado, as evidências empíricas encontradas relatam que a hipótese da existência de um prêmio por liquidez é válida apenas dentro do mercado acionário norteamericano. 
As constatações feitas para o mercado americano confirmam a hipótese de que ativos pouco líquidos, que tendem a possuir um custo de transação maior em suas negociações, apresentam retornos superiores. Os custos de transação associados à baixa liquidez são compensados pelo premio de liquidez, ou seja, a menor liquidez dos ativos está associada a uma maior rentabilidade. Tal associação se mostrou coerente com o tradicional pensamento financeiro de que investidores avessos ao risco exigem maiores retornos esperados como compensação pelo risco superior.

Por outro lado, quando observado o mercado acionário brasileiro, constatou-se que investidores que aplicam em ações com menor liquidez não recebem uma remuneração adicional por negociarem ativos que possuem um maior risco. Pelo contrário, estariam recebendo uma remuneração adicional ao empregar seus recursos em ações mais líquidas, que possuem um menor custo de transação e, consequentemente, são menos arriscadas.

Dado que a liquidez pode estar funcionando como um fator de risco na precificação dos ativos em mercados emergentes, pode-se conjecturar como hipótese explicativa para esse fenômeno que aumentos na intensidade de negociação das ações podem estar sendo vistos por investidores de mercados emergentes como aumentos na volatilidade das negociações. Desse modo, aumentos na volatilidade das ações indicariam um maior risco, levando as ações a oferecer um maior retorno. Nesse sentido, tais relacionamentos divergiriam dos encontrados em mercados eficientes e desenvolvidos, como o norte-americano, no qual os aumentos da liquidez são entendidos como um indicativo de redução no risco dos investimentos.

Adicionalmente, destaca-se a importância da utilização de métodos alternativos, que captem o comportamento dinâmico das séries financeiras que costumam apresentar uma evolução não linear, além de maior sensibilidade a crises econômicas. Ademais, sugere-se que sejam realizados estudos em outros países emergentes para verificar se permanece a relação encontrada no presente estudo.

\section{Referências}

Altinsoy, G. (2009). Time varying beta estimation for Turkish real estate investment trusts: An analysis of alternative modeling techniques. Thesis, Middle East Technical University, Ancara, Turquia.

Amihud, Y. (2002). Iliquidity and stock returns: Cross-section ant time series effects. Journal of Financial Markets, 5(1), 31-56.

Amihud, Y., \& Mendelson, H. (1986). Asset pricing and the bid-ask spread. Journal of Financial Economics, 17(2), 223-250.

Amihud, Y., \& Mendelson, H. (1988). Liquidity and asset price: Financial management implications. Financial Management, 17(1), 5-15.

Amihud, Y., \& Mendelson, H. (1991). Liquidity, assets prices and financial policy. Financial Analysts Journal, 47(6), 56-66.

Amihud, Y., \& Mendelson, H. (2006). Stockandbondliquidityanditseffectonpricesand financial policie. Financial Market Portfolio Management, 20(1), 19-32.

Antoniazzi, H. U. (2013). Formador de mercado e seu impacto nos custos de transação no mercado de ações brasileiro. Dissertação, Escola de Economia de São Paulo, Fundação Getúlio Vargas, São Paulo, Brasil.

Bouyé, E., Durrleman, V., Nikeghbali, A., Riboulet, G., \& Roncalli, T. (2000). Copulas for Finance: A Reading Guide and Some Applications. doi: 10.2139/ssrn.1032533

Coase, R. H. (1937). The nature of the firm. Economica, (4), 386-405.

Correia, L. F., \& Amaral, H. F. (2012). A influência da liquidez das ações sobre o retorno no mercado acionário brasileiro. Trabalho apresentado no Décimo Segundo Encontro Brasileiro de Finanças, São Paulo. Recuperado de: http://bibliotecadigital.fgv.br/ocs/index.php/ebf/12EBF/schedConf/presentations

Datar, V. T., Naik, N. Y., \& Radcliffe, R. (1998). Liquidity and stock returns: An alternative test. Journal of Financial Markets, 1(2), 203-219. 
Fagundes, J. (1998). Economia institucional: Custos de transação e impactos sobre política de defesa da concorrência. Revista de Economia Contemporânea, 2(1).

Fama, E. F., \& French, K. R. (2004). The capital asset pricing model: Theory and evidence.Journalof Economic Perspectives, 18(3), 25-46.

Gastineau,G.L., \&Kritzman,M.P. (1999).DicionáriodeAdministraçãode risco financeiro.SãoPaulo:BMFBrasil.

Jacobides,M. G., \&Winter,S. G. (2005).Theco-evolutionofcapabilitiesandtransactioncosts:

Explainingtheinstitutionalstructureofproduction.StrategicManagementJournal,26(5),395-413.

Jacoby, G., Fowler, D. J., \& Gottesman, A. A. (2000). The capital asset pricing model andtheliquidityeffect: A theoreticalapproach.JournalofFinancialMarkets,3(1),69-81.

Jondeau, E., \& Rockinger, M. (2006). The Copula-GARCH model of conditional dependencies: An international stock market application. Journal of International Money and Finance, 25(5), 827-853.

Jun, S., Marathe, A., \& Shawky, H. (2003). A liquidity and stock returns in emerging markets. Emerging Markets Review,4(1), 1-24.

Kim, D. (1993). The extent of non-stationarity of beta. Review of Quantitative Finance and Accounting, 3(1), 241-254.

Jondeau, E., Poon, S-H., \& Rockinger, M. (2007). Financial Modeling Under Non-Gaussian Distributions. London: Springer.

Lintner, J. (1965). The valuation of risk assets and selection of risky investments in stock portfolios and capital budgets. Review of Economics and Statistics, 47(1),13-37.

Liu, W. (2006). A liquidity-augmented capital asset pricing model. Journal of FinancialEconomics, 82(3), 631-671.

Ljung, G. M., \& Box, G. E. P. (1978). On a measure of lack of fit in time series models. Biometrika,65(2), 297-303.

Machado, M. A. V., \& Medeiros, O. R. (2012). Existe o efeito liquidez no mercado acionário brasileiro? Brazilian Business Review, 9(4), 30-42.

Majewski, Y. E. V., Minardi, A. M. A. F., \& Martins, S. R. (2014). Incorporação do prêmio por iliquidez no custo de capital próprio de empresas privadas no Brasil. Trabalho apresentado no Trigésimo Oitavo Encontro da ANPAD, Rio de Janeiro. Recuperado de: http://www.anpad.org.br/admin/pdf/2014_ EnANPAD_FIN36.pdf

Milgrom, P. R., \& Roberts, J. (1992). Economics, organizations and management. Englewood Cliffs, N.J.: Prentice Hall.

Minardi,A.M.,Sanvicente,A.Z., \&Monteiro,R. (2005).Spreaddecompraevendano mercado acionário brasileiro, liquidez, assimetria de informação e prêmio por liquidez. Trabalho apresentado no quinto Encontro Brasileiro de Finanças, Rio de Janeiro. Recuperado de: http://bibliotecadigital.fgv.br/ocs/ index.php/ebf/4EBF/paper/view/1483

Pastor, L., \& Stambaugh, R. F. (2003). Liquidity risk and expected stock returns. Journal of Political Economy, 111(3), 642-685.

Righi, M. B., \& Ceretta, P. S. (2012). Global risk evolution and diversification: A copula-DCC-GARCH Model Approach. Brazilian Review of Finance, 10(4).

Sanvicente, A. Z. (2011). Custos totais de transação no mercado secundário de ações no Brasil. Trabalho apresentado no Trigésimo Quinto Encontro da ANPAD, Rio de Janeiro. Recuperado de: http://www. anpad.org.br/admin/pdf/FIN185.pdf

Sharpe, W. F. (1964). Capital asset prices: A theory of market equilibrium under conditions of risk. Journal of Finance, 19(3), 425-442. 
Sunder, S. (1980). Stationarity of market risk: Random coefficients tests for individual stocks. Journal of Finance,35(4), 883-897.

Thielmann, R. (2013). A teoria dos custos de transação e as estruturas de governança: Uma análise do caso do setor de suinocultura no Vale do Rio Piranga - MG. Trabalho apresentado no Décimo Simpósio de Excelência em Gestão e Tecnologia, Rio de Janeiro, Brasil. Recuperado de: http://www. aedb.br/seget/arquivos/artigos13/991852.pdf

Tsay, R. S. (2010). Analysis of financial time series (3a ed). Hoboken: John Wiley \& Sons.

Vieira, K. M., Silveria, V. G., \& Righi, M. B. (2012). Análise do comportamento temporal da liquidez no mercado acionário brasileiro. Revista de Finanças Aplicadas, 1(3), 1-18.

Williamson, O. E. (1985). Vertical integration: Theory and policy. In O. E. Williamson, The Economiclnstitutions of Capitalism (cap.4). New York: The Free Press.

Zhou, J. (2013). Conditional market beta for REITs: A comparison of modeling techniques. Economic Modelling, 30(1), 196-204.

Submetido em: 04/07/2019

Aprovado em: 05/02/2019 